\title{
MODELAGEM DO INCREMENTO EM DIÂMETRO DA Araucaria angustifolia EM UMA FLORESTA OMBRÓFILA MISTA NO CENTRO- SUL DO PARANÁ
}

\author{
Thiago Floriani Stepka ${ }^{1}$, Andrea Nogueira Dias ${ }^{2}$, Afonso Figueiredo Filho ${ }^{3}$, \\ Sebastião do Amaral Machado ${ }^{4}$, Alex Roberto Sawczuk ${ }^{5}$ \\ ${ }^{1}$ Eng. Florestal, Dr., UNICENTRO, Irati, PR, Brasil - tfstepka@ yahoo.com.br \\ ${ }^{2}$ Eng. Florestal, Dra ${ }^{\text {. }}$, Depto. de Engenharia Florestal, UNICENTRO, Irati, PR, Brasil - andias@ irati.unicentro.br \\ ${ }^{3}$ Eng. Florestal, Dr., Depto. de Engenharia Florestal, UNICENTRO/UFPR, PR, Brasil - afigfilho@ gmail.com \\ ${ }^{4}$ Eng. Florestal, Dr., Depto. de Ciências Florestais, UFPR, Curitiba, PR, Brasil - samachado@ufpr.br \\ ${ }^{5}$ Eng. Florestal, M.Sc., Depto. de Extensão Florestal, IEF, Amapá, AP, Brasil - alex.sawczuk@ yahoo.com.br
}

Recebido para publicação: 18/06/2011 - Aceito para publicação: 15/03/2012

\begin{abstract}
Resumo
O objetivo deste trabalho foi testar a hipótese sobre a viabilidade em ajustar modelos capazes de estimar o incremento em diâmetro para a Araucaria angustifolia que ocorre naturalmente em uma Floresta Ombrófila Mista na Floresta Nacional de Irati, estado do Paraná, a partir de diversas tentativas de estratificação dos dados. Na floresta mencionada foram instaladas e medidas, em 2002, 25 parcelas permanentes de um hectare cada $(100 \mathrm{~m}$ x $100 \mathrm{~m})$, as quais foram remedidas em $2005 \mathrm{e}$ 2008. Todas as árvores com diâmetro á altura do peito (DAP) maior ou igual a $10 \mathrm{~cm}$ foram numeradas, medidas e identificadas. 1.039 árvores de araucária presentes nas 3 medições foram consideradas no ajustamento das equações, gerando-se valores de incremento médio anual dos três períodos de medição que serviram de base para o ajuste de 10 modelos matemáticos. Com a estratificação dos dados, em alguns casos, houve melhoras expressivas nos ajustes dos modelos, obtendo-se resultados mais significativos quando utilizados os incrementos agrupados em classes de diâmetro. No entanto, em razão da grande variabilidade dos incrementos da espécie estudada, concluiu-se que, para as várias estratificações aplicadas, os modelos testados não se mostraram eficientes para estimar o incremento em diâmetro, rejeitando-se, portanto, a hipótese da pesquisa.

Palavras-chave: Araucária; crescimento em diâmetro; modelagem.
\end{abstract}

\begin{abstract}
Increment diameter models for Araucaria angustifolia from an Ombrophyllous Mixed Forest in the center-south of Paraná. This research aims to test the viability of the hypothesis of adjustment of models capable of predicting diameter increment for Araucaria angustifolia from an Ombrophyllous Mixed Forest in the National Forest of Irati, Parana State using several data stratification methods. In this forest, twenty-five permanent plots of one hectare each $(100 \mathrm{~m} \mathrm{x} 100 \mathrm{~m})$ were installed and measured in 2002, being remeasured in 2005 and 2008. All trees with Diameter at Breast Height (DBH) greater than or equal to $10 \mathrm{~cm}$ were numbered, measured and identified. 1039 Araucaria trees were used in the adjustment of 10 mathematical models. These araucaria trees were measured three times (2002, 2005 and 2008) and the annual periodic increment was determined. The results revealed that data stratification, in some cases, improved adjustments for the models, with more significant results with increments gathered in diameter classes. However, the great variability of the increments of the focused species revealed that the stratification data hypothesis cannot be improved, and should be cast aside.

Keywords: Araucaria; diameter growth; modeling.
\end{abstract}

\section{INTRODUÇÃO}

Os processos dinâmicos (crescimento, mortalidade e ingresso) de uma floresta são de grande importância, visto que o estudo desses parâmetros indica o crescimento e as mudanças ocorridas na composição e estrutura da floresta. Portanto, a predição confiável desses processos, ressaltando-se o 
crescimento e a produção, torna-se imprescindível na adoção de tratamentos e medidas silviculturais mais adequadas para o manejo florestal sob regime de rendimento sustentado (MENDONÇA, 2003).

De acordo com Vanclay (1994) e Prodan et al. (1997), entende-se por crescimento o aumento de dimensões de um ou mais indivíduos em uma floresta em um determinado período de tempo. Tais dimensões podem ser o diâmetro, a altura, o volume, a biomassa, a área basal etc. Já a produção refere-se às dimensões finais ao término de determinado período. Portanto, a produção é o crescimento acumulado, enquanto o crescimento é a taxa de produção.

As estimativas desses parâmetros são obtidas, principalmente, por meio de inventário florestal contínuo com parcelas permanentes, por períodos curtos ou longos, geralmente na Floresta Ombrófila Mista as remedições são realizadas em intervalos de 1 ano (DURIGAN, 1999; PIZATTO, 1999; BARTH FILHO, 2002; SANQUETTA et al., 2003; GOMES, 2005; MOSCOVICH, 2006) ou 3 anos (FIGUEIREDO et al., 2010). O estudo do crescimento permite o entendimento dos processos por meio dos quais ocorrem as mudanças, em níveis de espécies e povoamentos.

Segundo Husch et al. (1982), o crescimento das árvores é influenciado pelas características da espécie interagindo com o ambiente. Para Prodan et al. (1997), as influências ambientais incluem fatores climáticos (temperatura, vento, precipitação e insolação), fatores pedológicos (características físicas e químicas, umidade e microrganismos), características topográficas (inclinação e elevação) e competição (influência de outras árvores, sub-bosque e animais), sendo que a soma desses fatores exprime o conceito de qualidade de sítio.

Uma função preditiva para o incremento do diâmetro de árvores é fundamental para modelos de crescimento, bem como para outros modelos funcionais baseados no indivíduo (árvore) ou em classes de tamanho (ALDER, 1995).

Para Zeide (1989), a modelagem do crescimento de árvores sempre é relacionada com o diâmetro (DAP) das árvores, devido à facilidade de medição dessa variável, sua sensibilidade às mudanças ambientais e densidade do povoamento e o fato de ser fortemente relacionada com o tamanho da copa, massa da árvore ou volume do tronco. Já para Alder (1995), nas florestas tropicais, o incremento diamétrico pode ser predito empiricamente a partir do DAP da árvore ou da área basal, situação de competição da árvore ou povoamento e variáveis contínuas ou categóricas do sítio.

A predição do crescimento e da produção de povoamentos manejados e não manejados é essencial para a credibilidade de um plano de manejo sustentável, tendo em vista que as decisões de manejo são tomadas com base na taxa de crescimento e na produção que esses povoamentos podem alcançar de acordo com essa mesma taxa (FERREIRA, 1997). No Brasil tais informações são pouco utilizadas pelos órgãos competentes na aprovação de planos de manejo, muitas vezes pela insuficiência de resultados de pesquisas na área.

Nesse contexto, alguns trabalhos sobre a modelagem do incremento em função do diâmetro têm sido realizados em vários países, sendo que a maioria deles encontrou valores do $\mathrm{R}^{2}$ baixo e erro padrão de estimativa alto.

Vanclay (1991) desenvolveu uma função de incremento em diâmetro com seis coeficientes usando o DAP, qualidade de sítio, área basal e um índice de competição. O referido autor também testou vários critérios de agrupamento de 237 espécies da floresta de Queensland, na Austrália, concluindo que o agrupamento produz equações mais robustas do que quando usando espécies individuais, embora o valor do $R^{2}$ da equação seja mais elevado para as espécies não agrupadas $(0,51)$ do que para espécies agrupadas $(0,49)$.

Leech et al. (1991) consideraram ajuste alternativo para agregar espécies para o desenvolvimento de equações polinomiais de volume usando $T^{2}$ de Hotelling e análise de componentes principais. $\mathrm{O}$ resultado não foi muito produtivo, pois essa metodologia somente deveria ser utilizada quando a ordem do polinômio e o sinal do termo de maior ordem são as mesmas.

Condit et al. (1993) fizeram um estudo de crescimento em 50 hectares de espécies de rápido crescimento em uma floresta no Panamá. Para as 28 espécies estudadas, o ajuste de um modelo polinomial do segundo grau resultou em um coeficiente de determinação variando de 0,001 a 0,985 , e dentre os 56 conjuntos de dados, apenas dez tiveram $\mathrm{R}^{2}$ maior que 0,70 , refletindo a variação do crescimento nas classes de DAP.

Chai e Lemay (1993) desenvolveram um modelo diamétrico para as florestas de Sarawak, Malásia, utilizando as variáveis independentes DAP, DAP ${ }^{2}$, índice de competição, idade desde a exploração, área basal, número de árvores, DAP quadrático e posição da copa. Para esse modelo com 
nove coeficientes, o $\mathrm{R}^{2}$ aj variou de 0,08 a 0,48 , sendo que a modelagem por espécie causou uma redução de $11,2 \%$ no erro padrão de estimativa quando comparado com o grupo de espécies.

Spathelf e Durlo (2001) empregaram um modelo polinomial de segundo grau para ajustar o incremento diamétrico com o centro de classe como variável independente em floresta secundária subtropical no estado do Rio Grande do Sul. O coeficiente de determinação obtido foi de 0,23, sendo o baixo valor atribuído à alta variação de incremento nas duas classes de menor tamanho.

Palahí et al. (2003) usaram um modelo de incremento diamétrico para a modelagem de crescimento de árvores de Pinus sylvestris na Espanha usando o DAP, um índice de competição, índice de sítio, área basal e idade do povoamento. $\mathrm{O} \mathrm{R}^{2}$ obtido foi de 0,24 , com erro padrão de estimativa de $64,1 \%$.

Palahí e Grau (2003) empregaram o DAP, índice de competição e idade do povoamento, para estimar o incremento de Pinus nigra no norte da Espanha, obtendo $\mathrm{R}^{2}$ de 0,14 e erro padrão de estimativa de $67,7 \%$, usando dados de incremento com intervalo de medição de cinco anos.

Phillips et al. (2004) aplicaram um modelo de incremento individual na floresta amazônica brasileira. O referido modelo tinha como variáveis independentes o DAP e um índice de competição, que resultou em um $\mathrm{R}^{2}$ variando entre 0,033 e 0,186 , conforme o grupo de espécies.

Liang et al. (2005) usaram um modelo de incremento diamétrico com o DAP, área basal e índice de sítio como variáveis independentes para florestas mistas de coníferas na Califórnia, obtendo um $\mathrm{R}^{2}$ de 0,25 para coníferas e 0,04 para folhosas.

Nebel e Meilby (2005) empregaram uma equação para modelar o incremento diamétrico para oito espécies na Amazônia peruana tendo como variáveis independentes o DAP e um índice de competição. Para o modelo testado, o coeficiente de determinação variou de 0,13 a 0,45 para as espécies estudadas, sendo que para uma delas não houve ajuste do modelo.

Namaalwa et al. (2005) desenvolveram um modelo de crescimento para as florestas áridas de Uganda que tinha como variáveis independentes o DAP, a área basal e a porcentagem da área basal pertencente a cada estrato da floresta. $\mathrm{O} \mathrm{R}^{2}$ variou de 0,03 a 0,08 de acordo com o estrato da floresta em análise.

Rossi (2007) utilizou modelos lineares e não lineares para realizar a modelagem do incremento em uma Floresta Ombrófila Mista em São João do Triunfo, Paraná. Utilizou dados de incremento periódicos anuais de intervalos de medição de 1 a 4 anos, sendo realizados ajustes para todas as espécies da floresta e somente para a araucária, alcançando valores de $\mathrm{R}^{2}$ de 0,5 a 0,8 e Syx de 8 a $40 \%$. Os dados de 1 ou 2 anos de intervalo tiveram os melhores ajustes, que podem estar atribuídos a um mesmo padrão de crescimento pelo qual a floresta esteja passando, (1996-1997) ou (1997-1999) por exemplo, justificando assim o melhor desempenho da modelagem.

O baixo desempenho no ajuste desses modelos, muitas vezes, é o reflexo da variação existente entre os incrementos das diferentes árvores (variação biológica), ou seja, vários fatores podem estar interferindo na dinâmica de cada indivíduo, como, por exemplo, a variabilidade genética, diferenças de idades (normalmente o crescimento é maior nas árvores mais jovens), condições ambientais e mesmo a competição com outros indivíduos.

Considerando as dificuldades em modelar o incremento diamétrico de espécies florestais nativas, nesta pesquisa buscou-se testar a hipótese de que a estratificação dos dados de incremento pode melhorar modelos matemáticos para estimar o incremento em diâmetro da Araucaria angustifolia monitoradas em uma Floresta Ombrófila Mista na Floresta Nacional de Irati, estado do Paraná.

\section{MATERIAIS E MÉTODOS}

\section{Fonte de dados}

Os dados utilizados no trabalho são provenientes de 25 parcelas permanentes de um hectare cada (100 m x 100 m), instaladas e medidas em 2002 em uma área de Floresta Ombrófila Mista (1272,9 ha) localizada na Floresta Nacional de Irati, estado do Paraná. Segundo Disperati e Oliveira Filho (2006), a FLONA de Irati situa-se a aproximadamente $150 \mathrm{~km}$ a oeste de Curitiba, Paraná. Criada em 1942, com área de 3.495 hectares, a FLONA tem 36\% (1.272,9 hectares) de floresta nativa com predominância de araucária e com o sub-bosque rico em espécies folhosas, como canelas, imbuias e angico, entre outras. Trata-se de uma área protegida por mais de 50 anos, sem similar na região.

As parcelas permanentes foram subdivididas em 4 subparcelas de 0,25 ha $(50 \mathrm{~m} \times 50 \mathrm{~m}) \mathrm{e}$ finalmente, para facilitar a numeração e o mapeamento das árvores (distâncias X, Y), cada subparcela foi 
subdividida ainda em 5 faixas de controle de $10 \mathrm{~m}$ x $50 \mathrm{~m}(0,05 \mathrm{ha})$. Piquetes de concreto foram colocados a cada $50 \mathrm{~m}$ e piquetes de madeira foram alocados a cada $10 \mathrm{~m}$.

No ano de 2002 foi realizada a medição de todas as árvores existentes nos 25 ha com o DAP igual ou superior a $10 \mathrm{~cm}$. As parcelas foram remedidas nos anos de 2005 e 2008. Nessas ocasiões também foram realizadas avaliações qualitativas das árvores, como segue:

- Qualidade de copa: copa vigorosa (3), copa média (2) e copa suprimida (1);

- Qualidade de fuste: fuste reto (3), fuste levemente tortuoso (2) e fuste tortuoso (1);

- Estado fitossanitário: fitossanidade boa (3), fitossanidade média (2), fitossanidade ruim ou atacada por pragas, podridão do fuste etc. (1);

- Estrato vertical: estrato superior (3), estrato médio (2) e estrato inferior (1).

Na remedição de 2008, a área de pesquisa (25 ha) tinha 14.178 árvores ou 567,12 árvores.ha ${ }^{-1}$, representadas por 118 espécies e 45 famílias, sendo que somente a araucária tinha 1.058 árvores nos 25 ha ou 42,32 indivíduos.ha ${ }^{-1}$. A área de pesquisa tinha uma área basal de $30,1 \mathrm{~m}^{2} \cdot \mathrm{ha}^{-1}$. A araucária, espécie objeto deste estudo, tinha $7,81 \mathrm{~m}^{2} \cdot \mathrm{ha}^{-1}$, participando com $25,9 \%$ da área basal para todas as espécies presentes no local de estudo.

\section{Ajuste dos modelos}

O incremento diamétrico periódico anual foi calculado com base no crescimento em diâmetro dos indivíduos medidos nas três ocasiões $(2002,2005$ e 2008), ou seja, aquelas árvores que permaneceram vivas durante cada período estudado. Assim foram descritos os incrementos periódicos anuais ocorridos em 2002-2005 e 2005-2008 e do período entre 2002-2008, os quais foram calculados com as fórmulas:

$$
\begin{gathered}
I P_{d}=d_{2005-o u-2008}-d_{2002-o u-2005} \\
I P A_{d}=\frac{I P_{d}}{P}
\end{gathered}
$$

Em que: $d_{2005 \text { ou } 2008}=$ DAP no final do período de crescimento avaliado $(\mathrm{cm}) ;$

$d_{2002 \text { ou } 2005}=$ DAP no início do período de crescimento avaliado $(\mathrm{cm})$;

$P=$ intervalo de medição (3 ou 6 anos).

Foram testados 10 modelos (Tabela 1) de incremento (três lineares e sete não lineares) utilizados por Vanclay (1994), Alder (1995) e Rossi (2007). Para a realização dos ajustes, foi utilizado o Microsoft Excel $^{\circledR}$ com a função de análise de regressão nos modelos lineares e a função solver (algoritmo GRG2) para os modelos não lineares.

\section{Avaliação dos modelos}

A seleção dos modelos foi com base no maior coeficiente de determinação ajustado ( $R^{2}$ aj) e menor erro padrão da estimativa relativo $\left(\mathrm{S}_{\mathrm{yx}} \%\right)$. Para os melhores modelos foi construída a distribuição gráfica dos resíduos em percentagem, a fim de verificar possíveis tendências nas estimativas. Além disso, verificou-se a significância dos coeficientes ( $\mathrm{p}$-valor $\alpha \leq 0,05$ ) para cada ajuste realizado. Quando o modelo com o melhor desempenho da modelagem apresentou um ou mais coeficientes não significativos, estes foram descartados e foi processado um novo ajuste somente com os coeficientes significativos. Já quando todos os coeficientes do modelo não foram significativos, o modelo foi descartado.

Como tentativa para melhoria dos ajustes, foram testadas diferentes formas de estratificação dos dados. Uma estratificação foi com relação à separação dos dados de acordo com as classes de qualidade de copa, fuste, estado fitossanitário e estrato vertical, como segue:

- Qualidade de copa: copa vigorosa (3), copa média (2) e copa suprimida (1);

- Qualidade de fuste: fuste reto (3), fuste levemente tortuoso (2) e fuste tortuoso (1);

- Estado fitossanitário: fitossanidade boa (3), fitossanidade média (2), fitossanidade ruim ou atacada por pragas, podridão do fuste etc. (1);

- Estrato vertical: estrato superior (3), estrato médio (2) e estrato inferior (1). 
Tabela 1. Modelos de incremento.

Table 1. Diameter increment models.

\begin{tabular}{llc}
\hline $\mathbf{N}^{\mathbf{0}}$ & \multicolumn{1}{c}{ Modelo } & Autor \\
\hline 1. & $I_{d}=\beta_{0}+\beta_{1} D A P+\beta_{2} D A P^{2}$ & ALEMDAG (1978) \\
2. & $I_{d}=\beta_{0}(D M a x-D A P)^{\beta_{1}} D A P^{\beta_{2}}$ & VANCLAY (1989) \\
3. & $I_{d}=\beta_{0} D A P^{\beta_{1}}-D A P^{\beta_{2}}$ & BERTALANFFY \\
4. & $I_{d}=\beta_{0} D A P^{\beta_{1}} e^{\beta_{2} D A P}$ & $*$ \\
5. & $I_{d}=D A P\left(\beta_{0}-\left(\beta_{1} \ln D A P\right)\right)$ & $*$ \\
6. & $I_{d}=\beta_{0}\left(1-\left(e^{-\beta_{1} D A P}\right)^{\beta_{2}}\right)$ & $*$ \\
7. & $I_{d}=e^{\beta_{0}+\beta_{1}(1 / D A P)}$ & $*$ \\
8. & $I_{d}=\beta_{0}+\beta_{1} D A P$ & $*$ \\
9. & $I_{d}=\beta_{0}+\beta_{1} D A P$ & $*$ \\
10. & $I_{d}=e^{\beta_{0}+\beta_{1} D A P}$ & $*$ \\
\hline
\end{tabular}

*Modelos ajustados por Rossi (2007). $I_{d}$ : incremento periódico anual médio do DAP (cm/ano); DAP: diâmetro a 1,3 m (cm) no início do período de crescimento; ln: logaritmo neperiano; $e$ : base do logaritmo neperiano; $\beta_{0,} \beta_{1,} \beta_{2,} \beta_{3}$ : coeficientes a ajustar.

Pelo fato de a araucária apresentar poucos indivíduos no estrato 1 (inferior) e 2 (médio), os mesmos foram agrupados para efeito de ajuste. Igual procedimento foi utilizado em relação à classe de qualidade de fuste, porque a araucária apresentou poucos indivíduos com fuste 1 (tortuoso) e 2 (levemente tortuoso).

Além disso, foram ajustados os modelos para cada classe diamétrica, com intervalos fixos de $10 \mathrm{~cm}$, como segue: $10-20 \mathrm{~cm}, 20-30 \mathrm{~cm}, \ldots, 80-90 \mathrm{~cm}$ e maior que $90 \mathrm{~cm}$ de diâmetro. Também foi testado o ajuste com dados de incremento periódico anual agrupado em classes de DAP, e nesse caso somente os dados do incremento periódico anual de 2002-2008 foram utilizados. O agrupamento foi realizado por parcela, considerando-se parcelas de 0,25 ha $(50 \mathrm{~m} \mathrm{x} 50 \mathrm{~m})$, tendo-se, portanto, 100 observações (parcelas).

\section{RESULTADOS E DISCUSSÕES}

\section{Ajuste para dados sem estratificação}

$\mathrm{Na}$ tabela 2 estão os resultados dos ajustes realizados para a araucária sem estratificação. Observa-se que, para o ajuste realizado com todos os dados (sem estratificação), o modelo 4 foi o que apresentou melhor $\mathrm{R}^{2}$ ajustado, igual a 0,1194 , e erro padrão de estimativa de $65,25 \%$. Tais resultados indicam um baixo desempenho do ajuste, resultado da grande variabilidade dos incrementos individuais.

Tabela 2. Estatísticas e coeficientes dos modelos de incremento em diâmetro ajustados para a araucária sem estratificação dos dados.

Table 2. Statistics and regression coefficients of the diameter annual increment models adjusted for the araucaria without stratification.

\begin{tabular}{|c|c|c|c|c|c|c|c|c|c|c|c|}
\hline \multirow{2}{*}{$\begin{array}{l}\text { Número de } \\
\text { observações }\end{array}$} & \multirow{2}{*}{ Estatísticas } & \multicolumn{10}{|c|}{ Modelo } \\
\hline & & 1 & 2 & 3 & 4 & 5 & 6 & 7 & 8 & 9 & 10 \\
\hline \multirow{5}{*}{3117} & Syx $\%$ & 65,66 & 65,94 & 65,98 & 65,25 & 186,40 & 65,68 & 65,68 & 67,20 & 68,12 & 67,52 \\
\hline & $\mathrm{R}^{2}$ ajustado & 0,1084 & 0,1007 & 0,0998 & 0,1194 & 0,1084 & 0,1078 & 0,1078 & 0,0661 & 0,0405 & 0,0568 \\
\hline & $\beta_{0}$ & $0,0466^{*}$ & $0,0070 \mathrm{~ns}$ & $0,4233 * *$ & $0,0079 * *$ & $0,0427 * *$ & $0,563 \mathrm{~ns}$ & $-0,401 * *$ & $0,285 * *$ & $0,380 * *$ & $-11,11 * *$ \\
\hline & $\beta_{1}$ & $0,0171^{* *}$ & $0,4573 * *$ & $0,1187 * *$ & $14,280 * *$ & $0,0041^{* *}$ & $0,222 \mathrm{~ns}$ & $-131,4 * *$ & $0,004 * *$ & $0,001 * *$ & $0,0073 * *$ \\
\hline & $\beta_{2}$ & $-0,0001 * *$ & $0,6061 * *$ & $-0,458 * *$ & $-0,026^{* *}$ & -------- & $0,222 \mathrm{~ns}$ & ------- & ------- & -------- & -------- \\
\hline
\end{tabular}

ns: p-valor $>0,05 ; *$ p-valor $\leq 0,05 ; * *$ p-valor $\leq 0,01$.

Os erros padrões de estimativa, embora apresentem valores superiores a $60 \%$, foram semelhantes a ajustes encontrados por outros pesquisadores, como Palahí et al. (2003), que modelaram o incremento de Pinus sylvestris na Espanha com erro padrão de estimativa de 64,1\%; Palahí e Grau (2003), na estimativa do incremento diamétrico de Pinus nigra no norte da Espanha, encontraram um erro padrão de estimativa de $67,7 \%$. 
Já no que se refere ao coeficiente de determinação, valores próximos aos encontrados nesta pesquisa foram também obtidos por Condit et al. (1993), Chai e Lemay (1993), Spathelf e Durlo (2001), Palahí et al. (2003), Palahí e Grau (2003), Phillips et al. (2004), Liang et al. (2005), Nebel e Meilby (2005), Namaalwa et al. (2005). Porém quase todos esses autores ajustaram o incremento não somente em função do diâmetro, como também em função da área basal e índices de competição da floresta.

A distribuição dos resíduos do melhor ajuste (Modelo 4) pode ser observada na figura 1A, em que os desvios foram relativamente altos, mas os resíduos não apresentam qualquer tendência.

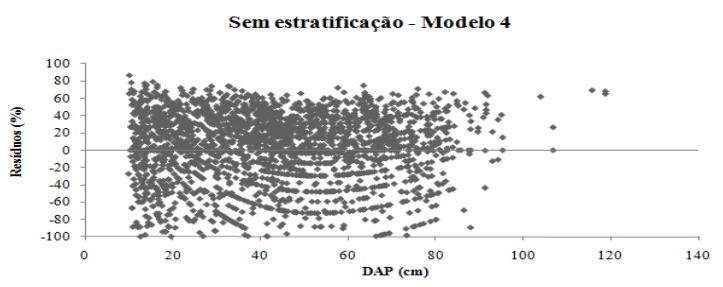

$\mathrm{C}$

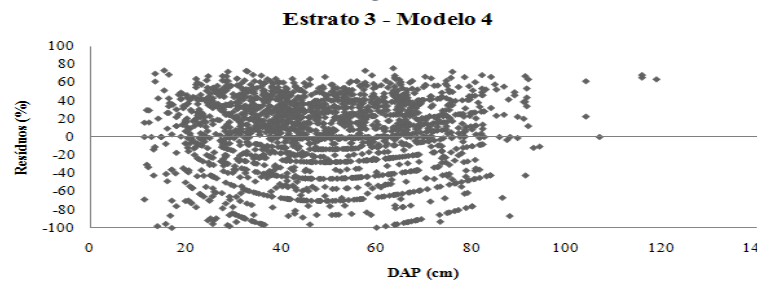

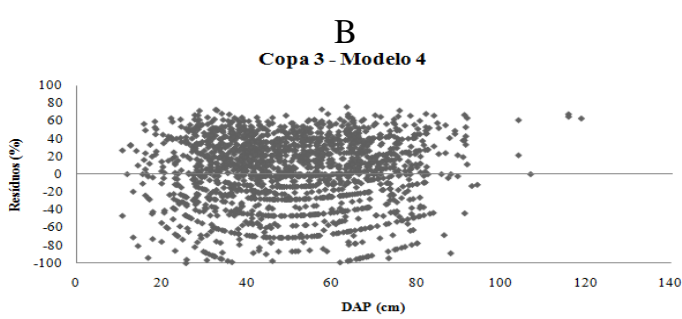

$\mathrm{D}$

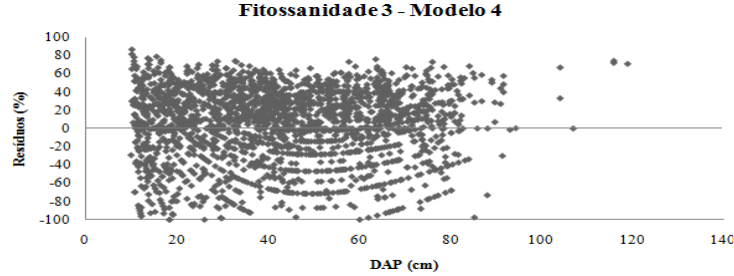

E

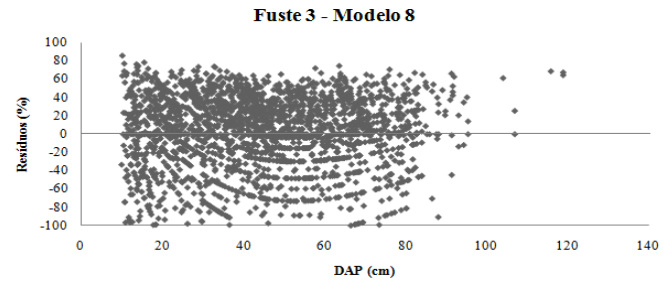

Figura 1. Distribuição dos resíduos dos melhores modelos para cada estratificação testada.

Figure 1. Residues distribution for the best models for each tested stratification.

\section{Ajuste por classes de qualidade da copa}

Na tabela 3 estão os resultados dos ajustes realizados para a araucária com dados estratificados por classe de qualidade de copa.

Constata-se que apenas para a classe de qualidade 3 obteve-se um pequeno ganho no ajuste, por meio do modelo 4 , que foi selecionado como o mais adequado (embora o valor do coeficiente $B_{0}$ tenha sido não significativo), com $\mathrm{R}^{2}$ ajustado de 0,0132 e erro padrão de $63,4 \%$. Deve ser ressaltado também que o maior número de observações utilizado nos ajustes em cada classe de qualidade pode ter influenciado positivamente a favor da classe de qualidade 3 , que a princípio dispõe de melhores condições de fotossíntese e consequentemente de crescimento.

Pode-se observar também, na tabela 3, vários modelos com $\mathrm{R}^{2}$ ajustado igual a zero. Gujarati (2000) relatou que quando o $\mathrm{R}^{2}$ é muito próximo de zero ou quando se recalcula o $\mathrm{R}^{2}$ para a variável de interesse, o $\mathrm{R}^{2}$ ajustado pode resultar em um valor negativo. Nesses casos, esse autor recomendou que os valores de $\mathrm{R}^{2}$ ajustados negativos sejam substituídos por zero. Esse fato ocorreu nos resultados apresentados nas tabelas 3 e 7, tendo sido adotada a recomendação de Gujarati (2000).

A distribuição dos resíduos apenas para o modelo de melhor desempenho na estratificação em classe de qualidade de copa está na figura 1B, constatando-se também que embora os desvios sejam amplos, os resíduos estão distribuídos sem qualquer tendência. 
Tabela 3. Estatísticas e coeficientes dos ajustes dos modelos de incremento em diâmetro para a araucária com estratificações por classes de copa.

Table 3. Statistics and regression coefficients of the diameter annual increment models adjusted for the araucaria stratified by tree crown classes.

\begin{tabular}{|c|c|c|c|c|c|c|c|c|c|c|c|}
\hline & \multirow{2}{*}{ Estatisticas } & \multicolumn{10}{|c|}{ Modelo } \\
\hline & & 1 & 2 & 3 & 4 & 5 & 6 & 7 & 8 & 9 & 10 \\
\hline \multirow{5}{*}{$\begin{array}{l}\text { Copa } 1 \\
\text { ( } 75 \text { obs.) }\end{array}$} & Syx\% & 87,30 & 87,34 & 87,32 & 87,33 & $\mathrm{NC}$ & 87,26 & 86,72 & 86,72 & 86,72 & 86,72 \\
\hline & $\mathrm{R}^{2}$ ajustado & 0 & 0 & 0 & 0 & $\mathrm{NC}$ & 0 & 0 & 0 & 0 & 0 \\
\hline & $\beta_{0}$ & $0,2041^{*}$ & $0,2057 \mathrm{~ns}$ & $11,257^{\text {** }}$ & $0,1292 \mathrm{~ns}$ & $\mathrm{NC}$ & $0,1823 \mathrm{~ns}$ & $-17,01^{* * k}$ & $0,1787 * *$ & 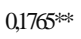 & $-17,214^{* * *}$ \\
\hline & $\beta_{I}$ & $-0,0023 \mathrm{~ns}$ & $-0,0146 \mathrm{~ns}$ & $0,3428^{\text {** }}$ & $0,1554 \mathrm{~ns}$ & $\mathrm{NC}$ & $0,4848 \mathrm{~ns}$ & $-0,532 \mathrm{~ns}$ & $-0,009 \mathrm{~ns}$ & $0,0001 \mathrm{~ns}$ & $-0,0007 \mathrm{~ns}$ \\
\hline & $\beta_{2}$ & $0,0001 \mathrm{~ns}$ & $-0,0363 \mathrm{~ns}$ & $0,3628^{\text {** }}$ & $-0,0069 \mathrm{~ns}$ & $\mathrm{NC}$ & $0,4848 \mathrm{~ns}$ & - & - & - & - \\
\hline \multirow{5}{*}{$\begin{array}{l}\text { Copa } 2 \\
(810 \text { obs.) }\end{array}$} & Syx \% & 82,14 & 82,27 & 82,18 & 82,06 & 82,14 & 82,13 & 82,05 & 82,95 & 83,96 & 83,43 \\
\hline & $\mathrm{R}^{2}$ ajustado & 0,0790 & 0,0761 & 0,0780 & 0,0807 & 0,0790 & 0,0791 & 0,0808 & 0,0607 & 0,0377 & 0,0498 \\
\hline & $B_{0}$ & $0,0441 \mathrm{~ns}$ & $0,0200 \mathrm{~ms}$ & $0,3947 \mathrm{~ns}$ & $0,0169 \mathrm{~ns}$ & $0,0357^{* * *}$ & $0,4953 \mathrm{~ns}$ & $-0,5552^{*}$ * & $0,1795^{* *}$ & $0,2648^{* *}$ & $-14,852^{\text {冰 }}$ \\
\hline & $B_{1}$ & $0,0159 *$ *k & $0,1949 \mathrm{~ns}$ & $0,1107 \mathrm{~ns}$ & $11,123^{* * k}$ & $0,0068^{* * *}$ & $0,2198 \mathrm{~ns}$ & 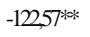 & $0,0056^{* *}$ & $0,0001^{* * k}$ & 0,0128 ** \\
\hline & $B_{2}$ & $-0,0002 * *$ & $0,6332 * *$ & $-0,4742 \mathrm{~ns}$ & $-0,0213^{* * *}$ & - & $0,2198 \mathrm{~ns}$ & - & - & - & - \\
\hline \multirow{5}{*}{$\begin{array}{l}\text { Copa } 3 \\
(2.232 \text { obs. })\end{array}$} & Syx \% & 63,65 & 63,73 & 63,73 & 63,43 & 63,53 & 63,58 & 63,70 & 63,86 & 63,87 & 63,86 \\
\hline & $\mathrm{R}^{2}$ ajustado & 0,0063 & 0,0037 & 0,0039 & 0,0132 & 0,0101 & 0,0085 & 0,0047 & 0 & 0 & 0 \\
\hline & $B_{0}$ & $0,3156^{* * * k}$ & $0,0552 \mathrm{~ns}$ & $0,7119^{* * *}$ & $0,0342 \mathrm{~ns}$ & $0,0519 * *$ & $0,5294 * *$ & $-0,5648^{* *}$ & $0,4961^{* *}$ & $0,5128^{* * k}$ & $-0,6984^{* * *}$ \\
\hline & $B_{l}$ & $0,0081^{\text {*k }}$ & $0,2930 \mathrm{~ns}$ & $-0,0387 \mathrm{~ns}$ & $0,9620^{\text {12k }}$ & $0,0105^{* *}$ & $0,2991^{\text {** }}$ & $-44,959$ ** & $0,0003 \mathrm{~ns}$ & $0,0001 \mathrm{~ns}$ & $0,0006 \mathrm{~ns}$ \\
\hline & $B_{2}$ & $-0,0001 * *$ & $0,2598 *$ & $-0,6047^{*}$ & $-0,0199 * *$ & - & $0,2991 \mathrm{~ns}$ & - & - & - & - \\
\hline
\end{tabular}

nc: ajuste que não convergiu; obs.: observações; ns: p-valor $>0,05$; *p-valor $\leq 0,05$; ** p-valor $\leq 0,01$.

\section{Ajuste por classes de posição vertical}

Na tabela 4 estão os dados dos ajustes realizados para a estratificação em classes de posição vertical. Tendo em vista o pequeno número de observações da classe 1, estas foram unidas com as observações da classe 2 para o ajuste dos modelos.

Tabela 4. Estatísticas e coeficientes dos ajustes dos modelos de incremento em diâmetro para a araucária com estratificações por classes de posição vertical.

Table 4. Statistics and regression coefficients of the diameter annual increment models adjusted for the araucaria stratified by vertical classes.

\begin{tabular}{|c|c|c|c|c|c|c|c|c|c|c|c|}
\hline & \multirow{2}{*}{ Estatísticas } & \multicolumn{10}{|c|}{ Modelo } \\
\hline & & 1 & 2 & 3 & 4 & 5 & 6 & 7 & 8 & 9 & 10 \\
\hline \multirow{5}{*}{$\begin{array}{l}\text { Estrato } 1,2 \\
\text { (648 obs.) }\end{array}$} & Syx \% & 84,15 & 84,10 & 84,11 & 84,11 & 84,14 & 84,17 & 84,08 & 71,19 & 84,34 & 84,18 \\
\hline & $\mathrm{R}^{2}$ ajustado & 0,0306 & 0,0316 & 0,0315 & 0,0314 & 0,0308 & 0,0300 & 0,0321 & 0,0317 & 0,0261 & 0,0298 \\
\hline & $B_{0}$ & $0,1310^{* * *}$ & $0,0775^{*}$ & $0,9938^{* * *}$ & $0,074 \operatorname{lns}$ & $0,0325^{* * *}$ & $0,3635^{* * k}$ & $-0,8731^{\text {* }}$ & $0,1495^{* *}$ & $0,2150^{\mathrm{k} *}$ & $-1,7105^{* * *}$ \\
\hline & $B_{1}$ & $0,0071^{*}$ & $-0,0163 \mathrm{~ns}$ & $0,1411 \mathrm{~ns}$ & $0,4121 \mathrm{~ns}$ & $0,0064^{* * *}$ & $0,2612^{* * k}$ & $-8,6482^{* * k}$ & $0,0055^{* *}$ & $0,0001^{\text {** }}$ & $0,0171^{* * k}$ \\
\hline & $\beta_{2}$ & $0,000 \mathrm{lns}$ & $0,4282^{* *}$ & $0,0747 \mathrm{~ns}$ & $0,0016 \mathrm{~ns}$ & --------- & $0,2612 \mathrm{~ns}$ & --------- & --------- & --------- & --------- \\
\hline \multirow{5}{*}{$\begin{array}{l}\text { Estrato } 3 \\
(2.469 \text { obs. })\end{array}$} & Syx \% & 64,70 & 64,78 & 64,80 & 64,45 & 64,54 & 64,61 & 64,74 & 65,03 & 65,09 & 65,04 \\
\hline & $\mathrm{R}^{2}$ ajustado & 0,0122 & 0,0097 & 0,0091 & 0,0200 & 0,0170 & 0,0151 & 0,0110 & 0,0021 & 0,0003 & 0,0019 \\
\hline & $\beta_{0}$ & $0,2632 * *$ & $0,0378 \mathrm{~ns}$ & $0,6396^{* *}$ & $0,0281^{*}$ & $0,0509^{1 * k}$ & $0,5286^{* 1 * k}$ & $-0,5409^{9 k k}$ & $0,4595 * *$ & $0,4907^{* * *}$ & $-0,767^{\text {冰 }}$ \\
\hline & $\beta_{I}$ & $0,0098^{* * ⿰ ㇇ ⿰ 亅 ⿱ 丿 丶 丶 ~}$ & $0,3265^{* * k}$ & $-0,0090 \mathrm{~ns}$ & $1,0241^{* *}$ & $0,0103^{* *}$ & $0,2852^{2 * k}$ & $-6,0527^{\text {**k }}$ & $0,0009 *$ & $0,0001 \mathrm{~ns}$ & $0,0017^{*}$ \\
\hline & $\beta_{2}$ & 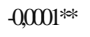 & $0,3186^{\text {冰 }}$ & $-0,5771^{* * k}$ & $-0,0209 \%$ & -------- & $0,2852 \mathrm{~ns}$ & --------- & --------- & --------- & --------- \\
\hline
\end{tabular}

ns: p-valor $>0,05$; obs.: observações; *p-valor $\leq 0,05 ; * *$ p-valor $\leq 0,01$.

O modelo 4 foi o que apresentou as melhores estatísticas para a estratificação da classe 3 , com $\mathrm{R}^{2}$ ajustado de 0,0200 e erro padrão de estimativa de 64,4\%. Já para o estrato 1 e 2, as melhores estatísticas foram geradas pelo modelo 7 , com o maior $\mathrm{R}^{2}$ ajustado $(0,0312)$, e pelo modelo 8 , que apresentou o menor erro padrão $(71,2 \%)$. Nessas estratificações por classes de estrato vertical, os melhores resultados de erro padrão de estimativa se encontram na classe 3, como também ocorreu na estratificação em classes de copa. Todavia, a estratificação não trouxe ganhos nos ajustes. A distribuição dos resíduos para o melhor modelo encontra-se na figura $1 \mathrm{C}$, com resultados similares aos resultados já apresentados para os casos anteriores. 


\section{Ajuste por classes de estado fitossanitário}

Os resultados dos ajustes realizados estratificando-se os dados por classes de estado fitossanitário estão na tabela 5. Os melhores coeficientes de determinação entre todas as estratificações testadas foram obtidos nessa estratificação, mas os erros médios foram piores que os ajustes sem estratificação. Na classe 3, o modelo 4 apresentou um $\mathrm{R}^{2}$ ajustado de 0,1048 e um erro padrão de estimativa de $67,4 \%$. Na classe 2, o modelo 7 apresentou um $\mathrm{R}^{2}$ ajustado de 0,1904 e um erro padrão de estimativa de $74,8 \%$. Na classe 1 , o melhor ajuste foi do modelo 2 , que apresentou um $\mathrm{R}^{2}$ ajustado de 0,2761 e um erro padrão de estimativa de $78,1 \%$. Dessa forma na estratificação pela fitossanidade, os maiores coeficientes de determinação ocorreram na classe 1. Já os menores erros padrões ocorreram na classe 3, embora alguns coeficientes dos modelos tenham sido não significativos. Na figura $1 \mathrm{D}$, pode-se observar que a distribuição dos resíduos, para a classe de estratificação 3, também se apresenta sem tendência.

Tabela 5. Estatísticas e coeficientes dos ajustes dos modelos de incremento em diâmetro para a araucária com estratificações por classes de estado fitossanitário.

Table 5. Statistics and regression coefficients of the diameter annual increment models adjusted for the araucaria stratified by phytohealth classes.

\begin{tabular}{|c|c|c|c|c|c|c|c|c|c|c|c|}
\hline & \multirow{2}{*}{ Estatísticas } & \multicolumn{10}{|c|}{ Modelo } \\
\hline & & 1 & 2 & 3 & 4 & 5 & 6 & 7 & 8 & 9 & 10 \\
\hline \multirow{5}{*}{$\begin{array}{l}\text { Fitossanidade } 1 \\
\text { (39 obs.) }\end{array}$} & Syx $\%$ & 81,77 & 78,19 & 82,16 & 80,20 & 81,02 & 82,01 & 81,36 & 81,12 & 82,22 & 82,25 \\
\hline & $\mathrm{R}^{2}$ ajustado & 0,2084 & 0,2761 & 0,2008 & 0,2385 & 0,2229 & 0,2038 & 0,2163 & 0,2210 & 0,1996 & 0,1991 \\
\hline & $\beta_{0}$ & $-0,0492 \mathrm{~ns}$ & $0,0233 \mathrm{~ns}$ & $0,9995^{\text {** }}$ & $0 \%$ & $0,0153 \mathrm{~ns}$ & $0,9623 \mathrm{~ns}$ & $-0,1530 \mathrm{~ns}$ & $0,0568 \mathrm{~ns}$ & $0,1650 \mathrm{hs}$ & $-1,8344^{* * *}$ \\
\hline & $\beta_{I}$ & $0,0134 \mathrm{~ns}$ & $0,0975 \mathrm{~ns}$ & $0,5195^{* * *}$ & $11,4847 * *$ & $0,0019 \mathrm{~ns}$ & $0,1043 \mathrm{~ns}$ & $-33,6935 \mathrm{~ns}$ & $0,002^{* *}$ & $0,0001^{* * *}$ & $0,0151^{\text {** }}$ \\
\hline & $\beta_{2}$ & $-0,0001 \mathrm{lns}$ & $0,6774 \mathrm{~ms}$ & $0,5059^{1 * k}$ & $-0,1495^{\text {** }}$ & . & $0,1043 \mathrm{~ns}$ & . . . . & - . . & - . . . & $\ldots$ \\
\hline \multirow{5}{*}{$\begin{array}{c}\text { Fitossanidade } 2 \\
\text { (147 obs.) }\end{array}$} & Syx $\%$ & 74,50 & 74,47 & 74,89 & 74,67 & 74,53 & 74,75 & 74,78 & 75,02 & 76,34 & 75,86 \\
\hline & $\mathrm{R}^{2}$ ajustado & 0,1964 & 0,1970 & 0,1881 & 0,1928 & 0,1957 & 0,1909 & 0,1904 & 0,1852 & 0,1562 & 0,1668 \\
\hline & $\beta_{0}$ & $0,0123 n s$ & $0,0213 \mathrm{~ns}$ & $0,8536^{*}$ & $0,0139 \mathrm{~ns}$ & $0,0290^{* *}$ & $0,6952 \mathrm{~ns}$ & $-0,2938^{*}$ & $0,1547^{1 * k}$ & $0,2660^{12 *}$ & $-1,4827^{* * *}$ \\
\hline & $\beta_{I}$ & $0,0156^{* *}$ & $0,0948 \mathrm{~ns}$ & $0,1886 \mathrm{~ms}$ & $1,0877^{*}$ & $0,0049^{* *}$ & $0,1617 \mathrm{~ns}$ & $-18,7127^{\text {冰 }}$ & $0,0063^{* *}$ & 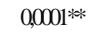 & $0,0135^{* *}$ \\
\hline & $\beta_{2}$ & $-0,000 \mathrm{lns}$ & $0,7242^{* *}$ & $0,0647 \mathrm{~ns}$ & $-0,0127 \mathrm{~ns}$ & - . . . & $0,1617 \mathrm{~ns}$ & - . . & - . . & $\ldots$ & $\ldots$ \\
\hline \multirow{5}{*}{$\begin{array}{l}\text { Fitossanidade } 3 \\
\text { (2.931 obs.) }\end{array}$} & Syx $\%$ & 67,90 & 68,14 & 68,32 & 67,42 & 67,88 & 67,97 & 68,03 & 69,50 & 70,28 & 69,75 \\
\hline & $\mathrm{R}^{2}$ ajustado & 0,0919 & 0,0855 & 0,0807 & 0,1048 & 0,0924 & 0,0901 & 0,0886 & 0,0486 & 0,0271 & 0,0417 \\
\hline & $\beta_{0}$ & $0,0539 *$ & $0,0056 \mathrm{~ms}$ & $0,4422^{\text {** }}$ & $0,0071^{* * *}$ & $0,0450^{1 \% *}$ & $0,5466 \mathrm{~ns}$ & $-0,4326^{* * *}$ & $0,3033^{* * k}$ & $0,3910^{1 * *}$ & $-1,0767^{\text {*** }}$ \\
\hline & $\beta_{I}$ & $0,0174^{* *}$ & $0,5183^{* *}$ & $0,1014^{* * k}$ & $1,4959 \%$ & $0,0088^{* * *}$ & $0,2339 \mathrm{~ns}$ & $-11,9794^{* k}$ & $0,0036^{1 * 2 *}$ & $0,0001^{* *}$ & $0,0066^{1 * k}$ \\
\hline & $\beta_{2}$ & $-0,0002^{* *}$ & $0,5961^{\text {*k }}$ & $-0,4778^{* *}$ & 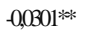 & - . . - & $0,2339 \mathrm{~ns}$ & - . . - & - . . . & - . - . - & - . . . \\
\hline
\end{tabular}

ns: p-valor $>0,05$; obs.: observações; $*$ p-valor $\leq 0,05 ; * *$ p-valor $\leq 0,01$.

\section{Ajuste por classes de qualidade do fuste}

Na tabela 6 estão os resultados dos ajustes dos modelos testados para os dados estratificados por classe de qualidade de fuste.

Da mesma forma que nas classes de posição do estrato vertical (poucas observações na classe 1), os indivíduos das classes 1 e 2 foram juntados para a realização da modelagem. Constata-se que, na classe 3 , o modelo 8 com $\mathrm{S}_{\mathrm{yx}} \%=55,3 \%$ e $\mathrm{R}^{2}$ ajustado de 0,0472 foi o que apresentou as melhores estatísticas. Já para as classes 1 e 2, o modelo 7 apresentou as melhores estatísticas, com um $\mathrm{R}^{2}$ ajustado de 0,1766 de erro padrão de $70,5 \%$. Da mesma forma que as demais estratificações, os resultados não foram satisfatórios. A distribuição dos resíduos encontra-se na figura $1 \mathrm{E}$.

Os ajustes para a menor classe de DAP $(10 \mathrm{a} 20 \mathrm{~cm})$ foram os piores, tanto no que diz respeito ao coeficiente de determinação ajustado quanto em relação ao erro padrão de estimativa, enquanto que os melhores resultados ocorreram nas classes 45 a $75(40 \mathrm{a} 80 \mathrm{~cm})$, sendo que os melhores desempenhos nessas classes foram do modelo 7, apresentando $\mathrm{R}^{2}$ ajustado de 0,1970, 0,0992, 0,1235 e 0,1487, e erro padrão de estimativa de 52,1,56,0,59,5 e 54,0\%, respectivamente, nas classes de 45, 55, 65 e $75 \mathrm{~cm}$ de diâmetro. Porém, na classe 75, o menor erro padrão de estimativa foi do modelo 8 , com 32,9\%, ou seja, a melhor precisão em todas as estratificações testadas. 
Constata-se que os incrementos diamétricos são mais homogêneos e melhor correlacionados com a variável DAP a partir da classe de $45 \mathrm{~cm}$, o que resultou em melhoria nos ajustes dos modelos. A distribuição dos resíduos dos melhores ajustes de cada classe de DAP está na figura 2. Observa-se uma grande variabilidade nos desvios, com dispersão até acima de $100 \%$. Nesse caso, não foi observada tendenciosidade nos resíduos, com exceção do observado no modelo 8 para a classe de 75, que, apesar de apresentar o menor erro padrão $(32,9 \%)$ apresentou uma tendência subestimativa nesse caso.

Tabela 6. Estatísticas e coeficientes dos ajustes dos modelos de incremento em diâmetro para a araucária com estratificações por classes de qualidade de fuste.

Table 6. Statistics and regression coefficients of the diameter annual increment models adjusted for the araucaria stratified by stem quality classes.

\begin{tabular}{|c|c|c|c|c|c|c|c|c|c|c|c|}
\hline & \multirow{2}{*}{ Estatísticas } & \multicolumn{10}{|c|}{ Modelo } \\
\hline & & 1 & 2 & 3 & 4 & 5 & 6 & 7 & 8 & 9 & 10 \\
\hline \multirow{5}{*}{$\begin{array}{c}\text { Fuste 1, } 2 \\
(222 \text { obs.) }\end{array}$} & Syx \% & 70,85 & 71,40 & 70,76 & 70,68 & 70,95 & 70,93 & 70,50 & 72,27 & 73,83 & 73,08 \\
\hline & $\mathrm{R}^{2}$ ajustado & 0,1686 & 0,1555 & 0,1707 & 0,1724 & 0,1661 & 0,1665 & 0,1766 & 0,1349 & 0,0970 & 0,1154 \\
\hline & $\beta_{0}$ & $0,0007 \mathrm{~ms}$ & $0,0405^{*}$ & $0,3382 \mathrm{~ns}$ & $0,0097 \mathrm{~ns}$ & $0,0311^{* * k}$ & $0,4764 \mathrm{~ns}$ & $-0,5890^{1 * k}$ &  & $0,2218^{\text {*1k }}$ & $-1,6627^{* * 1}$ \\
\hline & $\beta_{1}$ & $0,0172^{\text {** }}$ & $0,0402 \mathrm{~ns}$ & $0,1291 \mathrm{~ns}$ & $1,3011^{* *}$ & $0,0058^{* * 1 *}$ & $0,2088 \mathrm{~ns}$ & $-13,3722^{* *}$ & $0,0054^{* * *}$ & $0,0001^{\text {*k }}$ & $0,0144^{* *}$ \\
\hline & $\beta_{2}$ & $-0,0002^{2 * *}$ & $0,5752^{2 * k}$ & $-0,5095 \mathrm{~ns}$ & $-0,0259 *$ & ------- & $0,2088 \mathrm{~ns}$ & -------- & -------- & -------- & ------- \\
\hline \multirow{5}{*}{$\begin{array}{c}\text { Fuste } 3 \\
\text { (2.895 obs.) }\end{array}$} & Syx $\%$ & 64,97 & 65,23 & 65,26 & 64,59 & 64,90 & 64,96 & 65,01 & 55,28 & 66,89 & 66,46 \\
\hline & $\mathrm{R}^{2}$ ajustado & 0,0832 & 0,0759 & 0,0750 & 0,0940 & 0,0852 & 0,0838 & 0,0823 & 0,0470 & 0,0277 & 0,0410 \\
\hline & $\beta_{0}$ & $0,0729^{* * k}$ & $0,0100 \mathrm{~ns}$ & $0,4481^{* * *}$ & $0,0092 * *$ & $0,042^{* * k}$ & $0,5584 \mathrm{~ns}$ & $-0,4145^{* *}$ & $0,3167^{\text {**k }}$ & 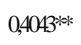 & $-1,0422^{* 1 * 1}$ \\
\hline & $\beta_{1}$ & $0,012^{\text {** }}$ & $0,4221^{* * *}$ & $0,103^{* * *}$ & $1,3800^{* *}$ & $0,0086^{1 * k}$ & $0,2301 \mathrm{~ns}$ & $-12,2649 \% *$ & $0,0034^{* * k}$ & $0,0001^{\text {*ak }}$ & $0,0063^{* * k}$ \\
\hline & $\beta_{2}$ & $-0,0001^{* *}$ & $0,5543^{* * k}$ & $-0,4701^{* *}$ & $-0,0261^{\text {*⿰冫 }}$ & ------- & $0,2301 \mathrm{ls}$ & -------- & -------- & -------- & -------- \\
\hline
\end{tabular}

ns: p-valor $>0,05$; obs.: observações; *p-valor $\leq 0,05$; ** p-valor $\leq 0,01$.

\section{Ajuste para cada classe de DAP}

A estratificação dos dados de incremento em diâmetro por classes de DAP também foi testada. Os resultados podem ser vistos na tabela 7 .

\section{Análise das estratificações}

Os resultados das estratificações nos ajustes dos modelos mostraram-se pouco eficientes, pois os valores do coeficiente de determinação ajustado obtidos sem estratificação variaram de 0,0400 a 0,1193, e, ao se estratificarem os dados, os melhores resultados foram para a classe de estado fitossanitário 1 e 2 , que apresentaram os maiores coeficientes de determinação ajustado, porém os menores valores do erro padrão foram encontrados na classe de fitossanidade 3. Apesar de alguns ganhos na estratificação dos dados, eles se mostraram poucos eficientes de maneira geral. Essa pouca qualidade nos ajustes também foi observada por Vanclay (1991). Nas estratificações realizadas, constatou-se certa superioridade do modelo 7, seguido dos modelos 3, 4, 5 e 8 .

\section{Ajuste para dados agrupados por classe de DAP}

Como os resultados das estratificações já relatadas não foram satisfatórios, ajustes dos modelos usando o incremento das árvores agrupadas por classe de diâmetro foram também testados. Assim, foram empregados incrementos periódicos anuais médios por classes de DAP do período 2002-2008 para a realização do ajuste. Nesse caso, cada parcela usada gerou um ponto para cada classe de DAP, tendo-se, portanto, 693 observações. Os resultados dos ajustes agrupados por classe de diâmetro encontram-se na tabela 8.

O coeficiente de determinação ajustado variou de 0,2546 a 0,3759 , e o erro padrão de estimativa variou de 38,62 a 42,20\%, bastante inferiores aos resultados até então encontrados nas estratificações testadas. $\mathrm{O}$ modelo 4, com um $\mathrm{R}^{2}$ ajustado de 0,3759 e um erro padrão de estimativa de $38,62 \%$, foi o que apresentou melhor desempenho. A distribuição dos resíduos do melhor resultado (modelo 4) para o ajuste do incremento por classe de diâmetro está na figura 2, sem tendências, exceto para a maior classe de DAP.

Spathelf e Durlo (2001) obtiveram um coeficiente de determinação de 0,23 para dados de floresta secundária no Rio Grande do Sul, ajustando o incremento diamétrico em função do centro de classe de DAP, sendo que o baixo valor foi atribuído pelos autores à alta variação de incremento nas duas classes de menor tamanho. 
Tabela 7. Estatísticas e coeficientes dos ajustes dos modelos de incremento para a araucária com estratificações para cada classe de DAP.

Table 7. Statistics and regression coefficients of the diameter annual increment models adjusted for the araucaria stratified by each dbh classes.

\begin{tabular}{|c|c|c|c|c|c|c|c|c|c|c|c|}
\hline & \multirow{2}{*}{ Estatísticas } & \multicolumn{10}{|c|}{ Modelo } \\
\hline & & 1 & 2 & 3 & 4 & 5 & 6 & 7 & 8 & 9 & 10 \\
\hline \multirow{5}{*}{$\begin{array}{l}\text { Classe } 15 \\
\text { (498 obs.) }\end{array}$} & Syx \% & 83,09 & 82,83 & 83,21 & 83,10 & 83,14 & 83,21 & 83,12 & 83,17 & 83,22 & 83,20 \\
\hline & $\mathrm{R}^{2}$ ajustado & 0,0139 & 0,0202 & 0,0112 & 0,0138 & 0,0129 & 0,0112 & 0,0133 & 0,0122 & 0,0090 & 0,0115 \\
\hline & $\beta_{0}$ & $-0,3162 \mathrm{~ns}$ & $0,0088 \mathrm{~ns}$ & $0,3978 n s$ & $0,0001 \mathrm{~ns}$ & $0,0335^{* *}$ & $0,3465 \mathrm{~ns}$ & $-0,9101^{* *}$ & $0,0948 \mathrm{~ns}$ & $0,1608^{* * *}$ & $-2,0472 *$ \\
\hline & $\beta_{1}$ & $0,0654 \mathrm{~ns}$ & $0,1473 n s$ & $0,0225 \mathrm{~ns}$ & $4,3070 \mathrm{~ns}$ & $0,0068^{*}$ & $0,2667 \mathrm{~ns}$ & $-8,5218^{* * *}$ & $0,00877^{* *}$ & $0,0003^{*}$ & $0,0368^{* *}$ \\
\hline & $\beta_{2}$ & $-0,0019 \mathrm{~ns}$ & $1,12292^{* * *}$ & $-0,6046 \mathrm{~ns}$ & $-0,2502 \mathrm{~ns}$ & - & $0,2667 \mathrm{~ns}$ & - & - & - & - \\
\hline \multirow{5}{*}{$\begin{array}{l}\text { Classe } 25 \\
\text { (423obs.) }\end{array}$} & Syx \% & 70,87 & 71,16 & 71,09 & 70,55 & 71,26 & $\mathrm{NC}$ & 70,92 & 71,12 & 71,17 & 71,08 \\
\hline & $\mathrm{R}^{2}$ ajustado & 0,0172 & 0,0092 & 0,0110 & 0,0260 & 0,0065 & $\mathrm{NC}$ & 0,0159 & 0,0103 & 0,0090 & 0,0113 \\
\hline & $\beta_{0}$ & $2,2932^{* *}$ & $0,9137 \mathrm{~ns}$ & $5,4362 \mathrm{~ns}$ & $66970928 \mathrm{~ns}$ & $0,0934 * *$ & $\mathrm{NC}$ & $-1,9212^{* *}$ & $0,5939 * *$ & $0,4663^{* *}$ & $-0,2955 \mathrm{~ns}$ \\
\hline & $\beta_{1}$ & $-0,1567^{*}$ & $0,0549 \mathrm{~ns}$ & $-0,8084 \mathrm{~ns}$ & $-8,5579 * *$ & $0,0248^{* * *}$ & $\mathrm{NC}$ & $20,0125^{* *}$ & $-0,0104 * *$ & $-0,0002^{*}$ & $-0,0324^{*}$ \\
\hline & $\beta_{2}$ & $0,0031^{*}$ & $-0,3327 \mathrm{~ns}$ & $-0,8084 \mathrm{~ns}$ & $0,3355 *$ & - & $\mathrm{NC}$ & - & - & - & - \\
\hline \multirow{5}{*}{$\begin{array}{l}\text { Classe } 35 \\
\text { (453 obs.) }\end{array}$} & Syx \% & 61,33 & 61,13 & 61,30 & 61,27 & 61,29 & $\mathrm{NC}$ & 61,20 & 61,27 & 61,27 & 61,26 \\
\hline & $\mathrm{R}^{2}$ ajustado & 0,0397 & 0,0459 & 0,0407 & 0,0416 & 0,0410 & $\mathrm{NC}$ & 0,0437 & 0,0418 & 0,0416 & 0,0420 \\
\hline & $\beta_{0}$ & 1,3906ns & $0,2133 \mathrm{~ns}$ & $72,7063 \mathrm{~ns}$ & $563256 \mathrm{~ns}$ & $0,1444^{* *}$ & $\mathrm{NC}$ & $-2,1068 * *$ & $1,2185^{* *}$ & $0,8630^{* * *}$ & $0,7338 * *$ \\
\hline & $\beta_{1}$ & $-0,0320 \mathrm{~ns}$ & $0,2274 \mathrm{~ns}$ & $-1,4200 \mathrm{~ns}$ & $-5,035 \mathrm{lns}$ & $0,0368^{* * *}$ & $\mathrm{NC}$ & $46,4601 * *$ & $-0,0215^{* *}$ & $-0,0003 * *$ & $-0,0430^{* * *}$ \\
\hline & $\beta_{2}$ & $0,0002 \mathrm{~ns}$ & 0,1353ns & $-1,4203 \mathrm{~ns}$ & 0,1108 ns & - & $\mathrm{NC}$ & - & - & - & - \\
\hline \multirow{5}{*}{$\begin{array}{l}\text { Classe } 45 \\
\text { (537 obs.) }\end{array}$} & Syx \% & 52,44 & 53,25 & 52,30 & 52,24 & 52,98 & $\mathrm{NC}$ & 52,13 & 52,79 & 52,93 & 52,38 \\
\hline & $\mathrm{R}^{2}$ ajustado & 0,1874 & 0,1621 & 0,1920 & 0,1936 & 0,1706 & $\mathrm{NC}$ & 0,1970 & 0,1766 & 0,1723 & 0,1895 \\
\hline & $\beta_{0}$ & $8,1287 * *$ & $0,3125 \mathrm{~ns}$ & $325988 \mathrm{~ns}$ & $34876281 \mathrm{~ns}$ & $0,2225 *$ & $\mathrm{NC}$ & $4,0925^{* *}$ & $2,4166^{* *}$ & $1,4748^{* * *}$ & $2,9795 *$ \\
\hline & $\beta_{1}$ & $-0,3099 * *$ & $0,4547 \mathrm{~ns}$ & $-3,3447 \mathrm{~ns}$ & $-5,2733^{* *}$ & $0,0557^{* *}$ & $\mathrm{NC}$ & $148,2027 * *$ & $-0,0434 * *$ & $-0,0005 * *$ & $-0,0833^{* * *}$ \\
\hline & $\beta_{2}$ & 0,0031 ** & $-0,0732 \mathrm{~ns}$ & $-3,5533 \mathrm{~ns}$ & $0,0422^{* * *}$ & - & $\mathrm{NC}$ & - & - & - & - \\
\hline \multirow{5}{*}{$\begin{array}{l}\text { Classe } 55 \\
\text { (408 obs.) }\end{array}$} & Syx \% & 56,2020 & 56,6170 & 56,1550 & 56,1250 & 56,3610 & $\mathrm{NC}$ & 56,0200 & 56,2930 & 56,3390 & 56,1510 \\
\hline & $\mathrm{R}^{2}$ ajustado & 0,0934 & 0,0799 & 0,0949 & 0,0958 & 0,0882 & $\mathrm{NC}$ & 0,0992 & 0,0904 & 0,0889 & 0,0950 \\
\hline & $\beta_{0}$ & $7,7834^{*}$ & $0,258 \mathrm{Ans}$ & $143314 \mathrm{~ns}$ & $15852042 \mathrm{~ns}$ & $0,1659^{* *}$ & $\mathrm{NC}$ & $-3,8139 * *$ & $2,0995 * *$ & $1,3073^{* *}$ & 2,4953 * \\
\hline & $\beta_{l}$ & $-0,2437 \mathrm{~ns}$ & $0,268 \mathrm{lns}$ & $-3,1555 \mathrm{~ns}$ & $4,7577^{*}$ & 0,0392 * * & $\mathrm{NC}$ & $167,0877^{* * *}$ & $-0,0296 * *$ & $-0,0003^{*} *$ & $-0,0593^{* *}$ \\
\hline & $\beta_{2}$ & $0,0020 \mathrm{~ns}$ & $0,0630 \mathrm{~ns}$ & $41366 n s$ & $0,0312 \mathrm{~ns}$ & - & $\mathrm{NC}$ & - & - & - & - \\
\hline \multirow{5}{*}{$\begin{array}{l}\text { Classe } 65 \\
\text { (399obs.) }\end{array}$} & Syx \% & 59,60 & 60,40 & 59,59 & 59,59 & 59,90 & $\mathrm{NC}$ & 59,46 & 59,82 & 59,88 & 59,57 \\
\hline & $\mathrm{R}^{2}$ ajustado & 0,1192 & 0,0956 & 0,1197 & 0,1197 & 0,1102 & $\mathrm{NC}$ & 0,1235 & 0,1129 & 0,1109 & 0,1200 \\
\hline & $\beta_{0}$ & $12,0264^{*}$ & $0,2794 \mathrm{~ns}$ & 11510984ns & $12010170 \mathrm{~ns}$ & $0,1784 * *$ & $\mathrm{NC}$ & $4,6939 * *$ & $2,6026^{* *}$ & $1,5602^{* *}$ & $3,4684 * *$ \\
\hline & $\beta_{1}$ & $-0,3322^{*}$ & $0,3054 \mathrm{~ns}$ & $-4,0827 \mathrm{~ns}$ & $4,1061^{* *}$ & $0,0410^{* * *}$ & $\mathrm{NC}$ & $254,0012 * *$ & $-0,0328 * *$ & $-0,0003^{*} *$ & $-0,0654 *$ \\
\hline & $\beta_{2}$ & $0,0024^{*}$ & $0,0255 \mathrm{~ns}$ & $-3256438 \mathrm{~ns}$ & 0,0009 ns & - & $\mathrm{NC}$ & - & - & - & - \\
\hline \multirow{5}{*}{$\begin{array}{l}\text { Classe } 75 \\
\text { (258 obs.) }\end{array}$} & Syx\% & 54,24 & 54,89 & 54,21 & 54,26 & 54,47 & $\mathrm{NC}$ & 54,03 & 32,93 & 54,45 & 54,14 \\
\hline & $\mathrm{R}^{2}$ ajustado & 0,1419 & 0,1213 & 0,1428 & 0,1412 & 0,1347 & $\mathrm{NC}$ & 0,1487 & 0,1373 & 0,1353 & 0,1452 \\
\hline & $\beta_{0}$ & $13,4896^{*}$ & $0,2410 \mathrm{~ns}$ & $50591748 \mathrm{~ns}$ & $0,4977 \mathrm{~ns}$ & $0,1845^{* *}$ & $\mathrm{NC}$ & $-5,2035 * *$ & $3,0102^{* *}$ & $1,7742^{* * *}$ & $4,0617^{* *}$ \\
\hline & $\beta_{1}$ & $-0,3233 n s$ & $0,3242 \mathrm{~ns}$ & $-4,2858 \mathrm{~ns}$ & $1,4539 \mathrm{~ns}$ & $0,0413^{* *}$ & $\mathrm{NC}$ & $331,7243^{* *}$ & $-0,0339$ ** & $-0,0002 * *$ & $-0,0645 *$ \\
\hline & $\beta_{2}$ & $0,0020 \mathrm{~ns}$ & $0,0565 *$ * & $-5463759 \mathrm{~ns}$ & $-0,0848 n \mathrm{~ns}$ & - & $\mathrm{NC}$ & - & - & - & - \\
\hline \multirow{5}{*}{$\begin{array}{l}\text { Classe } 85 \\
\text { (111 obs.) }\end{array}$} & Syx\% & 63,76 & 65,34 & 63,96 & 63,79 & 64,10 & $\mathrm{NC}$ & 63,25 & 64,02 & 64,09 & 63,47 \\
\hline & $\mathrm{R}^{2}$ ajustado & 0,1180 & 0,0736 & 0,1125 & 0,1171 & 0,1085 & $\mathrm{NC}$ & 0,1320 & 0,1107 & 0,1088 & 0,1261 \\
\hline & $\beta_{0}$ & $35,4413 \mathrm{~ns}$ & $0,1816 n s$ & 60691543ns & $0,3911 \mathrm{~ns}$ & $0,2236 \mathrm{~ns}$ & $\mathrm{NC}$ & $-9,1634 *$ & $4,0966 \mathrm{nns}$ & $2,2804 \mathrm{~ns}$ & $7,6327 \mathrm{~ns}$ \\
\hline & $\beta_{1}$ & $-0,8040 \mathrm{~ns}$ & $0,443 \mathrm{~ns}$ & $-4,0796^{* *}$ & $2,5112 \mathrm{~ns}$ & $0,0493 \mathrm{~ns}$ & $\mathrm{NC}$ & $692,234 * *$ & $-0,0437 \mathrm{~ns}$ & $-0,0003 n s$ & $-0,1018 \mathrm{~ns}$ \\
\hline & $\beta_{2}$ & $0,0046 \mathrm{~ns}$ & $0,0579^{* * *}$ & $-0,1843 n \mathrm{~ns}$ & $-0,1322 * *$ & - & $\mathrm{NC}$ & - & - & - & - \\
\hline \multirow{5}{*}{$\begin{array}{l}\text { Classe }>90 \\
\text { (30 obs.) }\end{array}$} & Syx \% & 54,45 & 56,21 & 57,16 & 56,39 & 56,12 & 57,15 & 56,23 & 56,12 & 56,00 & 56,00 \\
\hline & $\mathrm{R}^{2}$ ajustado & 0,0496 & 0 & 0 & 0 & 0 & 0 & 0 & 0 & 0 & 0 \\
\hline & $\beta_{0}$ & $14,2238 n s$ & $0,3512 \mathrm{~ns}$ & $0,9895 *$ & $2760000000 \mathrm{~ns}$ & $0,006 \mathrm{lns}$ & 1301,6ns & $0,4869 \mathrm{~ns}$ & $0,036 \mathrm{Ans}$ & $0,3174 n s$ & $-1,5181 \mathrm{~ns}$ \\
\hline & $\beta_{1}$ & $-0,2739 \mathrm{~ns}$ & $-0,040$ ns & $0,6735^{* *}$ & $-5,7872 * *$ & Ons & $0,0022 \mathrm{~ns}$ & $-94,557 \mathrm{~ns}$ & $0,0059 \mathrm{~ns}$ & $0,0001 \mathrm{~ns}$ & $0,0105 \mathrm{~ns}$ \\
\hline & $\beta_{2}$ & $0,0014 \mathrm{~ns}$ & $0,1393 n s$ & 0,6649 *** & $0,0672^{* *}$ & - & $0,0022 \mathrm{~ns}$ & - & - & - & - \\
\hline
\end{tabular}

nc: ajuste que não convergiu; ns: p-valor $>0,05$; obs.: observações; * p-valor $\leq 0,05$; ** p-valor $\leq 0,01$. 

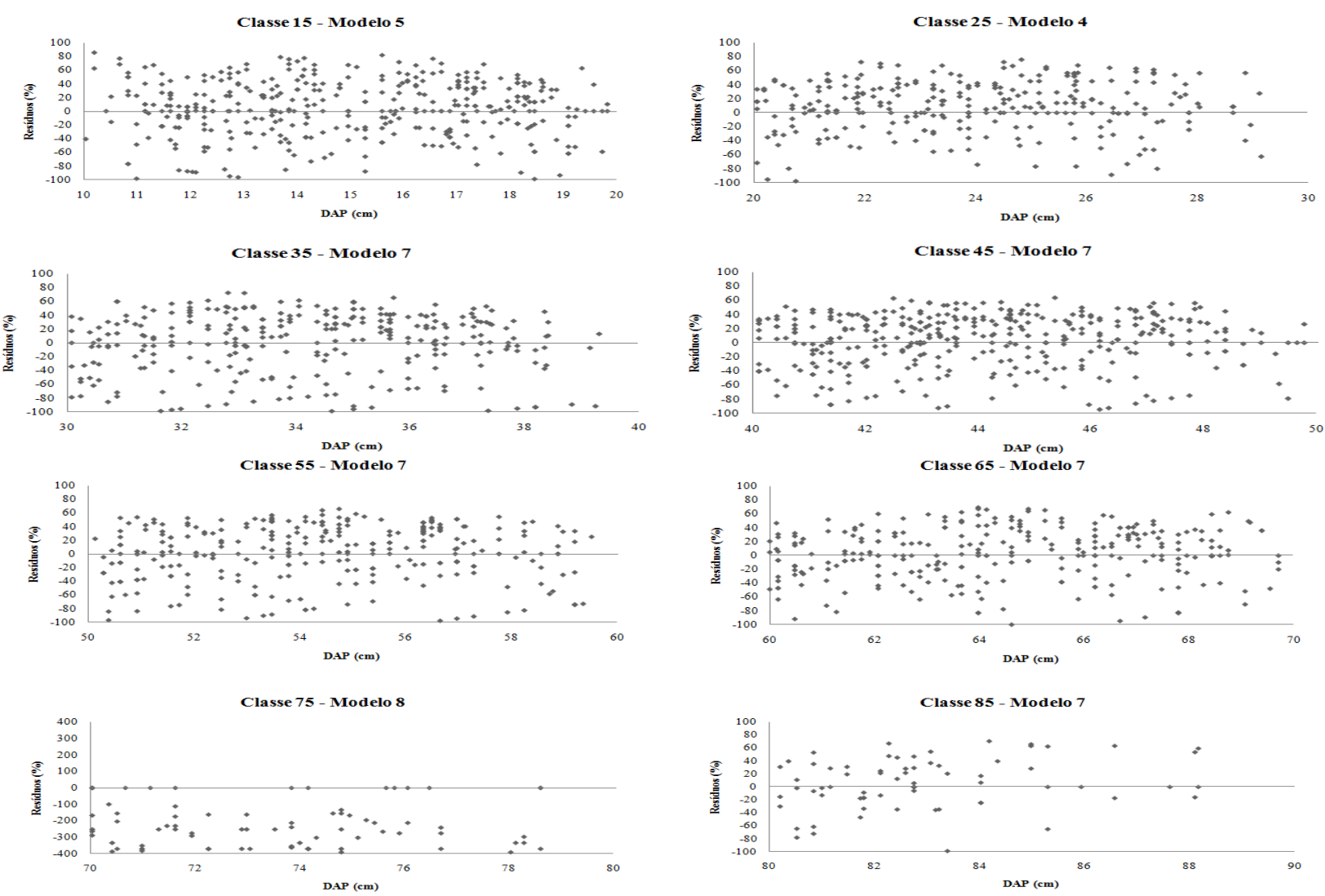

Classe $>90-$ Modelo 3



Figura 2. Distribuição dos resíduos para cada classe DAP.

Figure 2. Residues distribution by each dbh classes.

Tabela 8. Estatísticas e coeficientes dos ajustes dos modelos de incremento para a araucária com dados por classe de DAP.

Table 8. Statistics and regression coefficients of the diameter annual increment models adjusted for the araucaria with data by dbh classes.

\begin{tabular}{|c|c|c|c|c|c|c|c|c|c|c|c|}
\hline & \multirow{2}{*}{ Estatísticas } & \multicolumn{10}{|c|}{ Modelo } \\
\hline & & 1 & 2 & 3 & 4 & 5 & 6 & 7 & 8 & 9 & 10 \\
\hline \multirow{5}{*}{ (693 obs.) } & Syx $\%$ & 38,68 & 39,29 & 38,78 & 38,62 & 38,69 & 38,64 & 38,84 & 39,70 & 42,20 & 41,12 \\
\hline & $\mathrm{R}^{2}$ ajustado & 0,3739 & 0,3540 & 0,3707 & 0,3759 & 0,3737 & 0,3752 & 0,3695 & 0,3394 & 0,2546 & 0,2925 \\
\hline & $\beta_{0}$ & $0,0583^{* *}$ & $0,0002 \mathrm{~ns}$ & $0,1771^{* * *}$ & $0,0154^{* *}$ & $0,0248 * * *$ & $0,6375^{* *}$ & $-0,3669 * *$ & $0,1666^{* * *}$ & $0,2911^{* *}$ & $-1,4006^{* * *}$ \\
\hline & $\beta_{l}$ & $0,0100^{* *}$ & $0,8195^{\text {** }}$ & $0,2834 * *$ & $0,9695^{* *}$ & 0,0041 ** & 0,1537 *** & $-21,6551^{* *}$ & $0,0049^{* * *}$ & $3,8 \mathrm{E}-05^{* *}$ & 0,0097 ** \\
\hline & $\beta_{2}$ & $-4,9 \mathrm{E}-05^{* *}$ & $0,9740 * *$ & $-0,5947 * *$ & $-0,0086^{* *}$ & - & 0,1537 *** & - & - & - & - \\
\hline
\end{tabular}

ns: p-valor $>0,05$; obs.: observações; *p-valor $\leq 0,05$; ** p-valor $\leq 0,01$. 


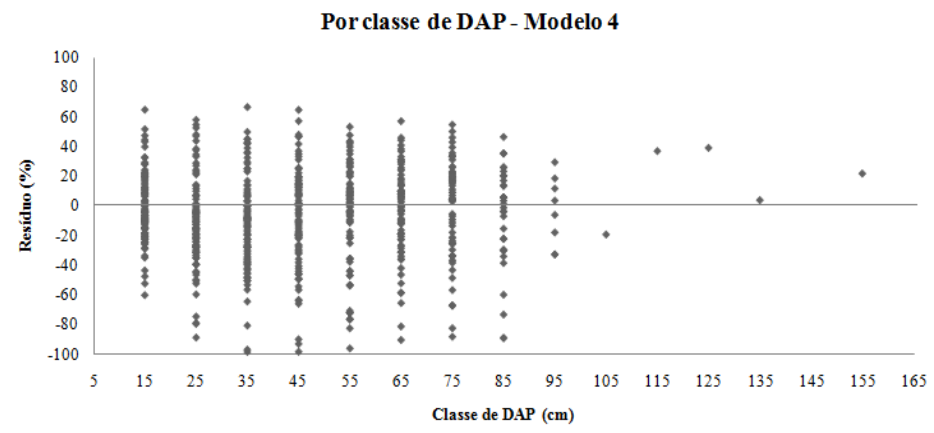

Figura 3. Distribuição dos resíduos para o melhor resultado do ajuste.

Figure 3. Residues distribution for the best result of the adjustment.

Rossi (2007) testou alguns modelos para o ajuste dos incrementos médios agrupados por classes de DAP e percebeu que os ajustes para a araucária de maneira geral apresentam melhores estatísticas do que os resultados obtidos no ajuste dos incrementos para todas as espécies. Esse autor constatou que, dos 168 casos testados, somente em 22 o erro padrão de estimativa foi inferior a $15 \%$ e em apenas um caso foi inferior a $10 \%$. Quanto ao coeficiente de determinação ajustado, houve variação de 0,00 a 0,87 , ocorrendo 22 casos com valores superiores a 0,80 . $\mathrm{O}$ autor citado modelou o incremento médio anual obtido em períodos de 1, 2, 3 e 4 anos de monitoramento, sendo que os melhores ajustes aconteceram com dados provenientes de períodos de monitoramento de 2 anos.

Algumas vezes, têm-se utilizado ajustes com dados médios agrupados por classe de diâmetro com uma única observação média do incremento para cada classe de DAP. As estatísticas do ajuste, nesse caso, obviamente apresentam coeficientes de determinação próximos a $100 \%$ e erros médios bastante baixos, porém não representam a realidade da variabilidade dos dados.

O baixo desempenho na modelagem do incremento diamétrico, utilizando como entrada o DAP no início do período de monitoramento, é explicado pela grande variabilidade dos incrementos das árvores em florestas nativas para um mesmo diâmetro inicial. Nessas florestas ocorre uma grande variação dos incrementos das árvores (variação biológica), muitas vezes relacionada a fatores genéticos, diferenças de idades entre as árvores, qualidade do solo e competição. Várias árvores que apresentam, por exemplo, $50 \mathrm{~cm}$ de diâmetro, podem apresentar um crescimento diferenciado entre elas devido a vários fatores que estão interferindo na dinâmica de cada indivíduo, ou seja, esse crescimento diferenciado das árvores de mesmas dimensões é refletido no baixo desempenho dos ajustes. A araucária, foco deste estudo, tinha em média 42 árvores por hectare, incremento médio anual de $0,45 \mathrm{~cm} / \mathrm{ano}$, variando de $0 \mathrm{a}$ $1,33 \mathrm{~cm} /$ ano, desvio padrão de $0,24 \mathrm{~cm}$ e um coeficiente de variação de 53,96\%.

Finalmente, os resultados desta pesquisa indicam que as estratificações testadas não viabilizaram a possibilidade de modelar os incrementos com um bom desempenho e dessa forma, alternativas deverão ser buscadas para a geração de modelos mais eficientes, como, por exemplo, a utilização de modelos com mais variáveis independentes como área basal ou índices de competição, testes com modelos ecofisiológicos e uma estratificação para diferentes níveis de densidade da floresta.

\section{CONCLUSÕES}

- A hipótese de que a estratificação dos dados poderia trazer melhorias nos ajustes dos modelos testados para estimar o incremento diamétrico da araucária foi rejeitada, pelo menos para os dados desta pesquisa.

- A modelagem para as árvores de araucária com melhores condições fitossanitárias (classe 3) apresentou as melhores estatísticas de todas as estratificações consideradas neste trabalho.

- Em algumas estratificações ocorreram melhoras na precisão, mas que ainda não são capazes de gerar estimativas adequadas para o incremento diamétrico da espécie estudada.

- Os ajustes realizados para os dados médios por classe de diâmetro apresentam melhorias substanciais nas estatísticas, porém, por serem ajustados com dados médios, não expressam a realidade da variabilidade do incremento diamétrico da araucária.

- A distribuição dos resíduos, apesar de apresentar percentuais elevados, não apresentou tendências nas estimativas, exceto nos ajustes médios por classe de DAP, com subestimativas do incremento na última classe de DAP. 


\section{REFERÊNCIAS}

ALDER, D. Growth modeling for mixed tropical forests. Tropical Forestry Papers, Oxford Forestry Institute, Department of Plant Sciences, University of Oxford, n. 30, 1995.

BARTH FILHO, N. F. Delineamentos de um sistema de monitoramento de crescimento e produção em campo para florestas naturais: aplicação na floresta com araucária. Curitiba, 2002. 86 p. Dissertação (Mestrado em Engenharia Florestal) - Curso de Pós-Graduação em Engenharia Florestal, Universidade Federal do Paraná.

CHAI, F. Y. C.; LEMAY, V. M. Development and testing of diameter increment models for mixed swamp forests of Sarawak. Forest Ecology and Management, Amsterdam, v. 58, p. 51 - 64. 1993.

CONDIT, R.; HUBBELL, S. P.; FOSTER, R. B. Indentifying fast-growing native trees from the Neotropics using data from a large, permanent census plot. Forest Ecology and Management, Amsterdam, v. 62, p. 123 - 143. 1993.

DISPERATI, A. A.; OLIVEIRA FILHO, P. C. Copas de araucária observadas em fotografias aéreas 70 mm colorido normal em escala muito grande (1:1000). In: Anais do VII Seminário de Atualização em Sensoriamento Remoto e Sistemas de Informações Geográficas Aplicados à Engenharia Florestal / Orgs. Attilio Antonio Disperati, João Roberto dos Santos. Curitiba, PR, de 17 a 19 de outubro de 2006. Curitiba, PR: FUPEF - Fundação de Pesquisas Florestais do Paraná, 2006.

DURIGAN, M. E. Florística, dinâmica e análise proteica de uma Floresta Ombrófila Mista em São João do Triunfo - PR. Curitiba, 1999. 125 p. Dissertação (Mestrado em Engenharia Florestal) - Setor de Ciências Agrárias, Universidade Federal do Paraná.

FERREIRA, R. L. C. Estrutura e dinâmica de uma floresta secundária de transição, Rio Vermelho e Serra Azul de Minas, MG. 208 f. Tese (Doutorado em Ciência Florestal) - Universidade Federal de Viçosa. Viçosa, 1997.

FIGUEIREDO FILHO, A.; DIAS, A. N.; STEPKA, T. F.; SAWCZUK, A. R. Crescimento, mortalidade, ingresso e distribuição diamétrica em Floresta Ombrófila Mista. Revista Floresta, Curitiba, PR, v. 40, n. 4, out./dez. 2010.

GOMES, J. F. Classificação e crescimento de grupos ecológicos na Floresta Ombrófila Mista da FLONA de São Francisco de Paula, RS. Santa Maria, 2005. 75 f. Dissertação (Mestrado em Ciências Florestais) - Programa de Pós-Graduação em Engenharia Florestal, Universidade Federal de Santa Maria.

GUJARATI, D. N. Econometria básica. São Paulo: MAKRON Books, 2000. 846 p.

HUSCH, B.; MILLER, C. I.; BEERS, T. W. Forest mensuration. 3 ed. New York: John Wiley \& Sons, 1982.

LEECH, J. W.; CORRELL, R. L.; MYINT, A. K. Use of principal-coordinate analysis to assist in aggregating species for volume-table construction. Forest Ecology and Management, Amsterdam, v. 40: p. 279 - 288. 1991.

LIANG, J.; BUONGIORNO, J.; MONSERUD, R. A. Estimation and application of a growth and yield models for uneven-aged mixed stands in California. International Forestry Review. v. 7, n. 2, p. 101 $112,2005$.

MENDONÇA, A. C. A. Caracterização e simulação dos processos dinâmicos de uma área de floresta tropical de terra firme utilizando matrizes de transição. $81 \mathrm{f}$. Dissertação (Mestrado em Engenharia Florestal) - Setor de Ciências Agrárias, Universidade Federal do Paraná. Curitiba, 2003.

MOSCOVICH, F. A. Dinâmica de crescimento de uma Floresta Ombrófila Mista em Nova Prata, RS. Santa Maria, 2006. 130 f. Tese (Doutorado em Ciências Florestais) - Programa de Pós-Graduação em Engenharia Florestal, Universidade Federal de Santa Maria. 
NAMAALWA, J.; EID, T.; SANKHAYAN, P. A multi-species density-dependent matrix growth model for the dry woodlands of Uganda. Forest Ecology and Management, Amsterdam, v. 213, p. 312 - 327, 2005.

NEBEL, G.; MEILBY, H. Growth and populations structure of timber species in Peruvian Amazon flood plains. Forest Ecology and Management, Amsterdam, v. 215, p. 196 - 211, 2005.

PALAHÍ, M.; GRAU. J. M. Preliminary site índex model and individual-tree growth and mortality models for Black pine (Pinus nigra Arn.) in Catalonia (Spain). Inves. Agrar.: Sist. Recur. For. v. 12, n. 1, p. 137 - 148, 2003.

PALAHÍ, M.; PUKKALA, T.; MIINA, J.; MONTERO, G. Individual-tree growth and mortality models for Scots pine (Pinus sylvestris L.) in north-east Spain. Ann. For. Sci. v. 60, p. 1 - 10, 2003.

PHILlIPS, P. D.; AZEVEDO, C. P.; DEGEN, B.; THOMPSON, I. S.; SILVA, J. N. M.; VAN GARDINGEN, P. R. An individual-based spatially explicit simulation model for strategic forest management planning in the eastern Amazon. Ecological Modeling, Amsterdam, v. 173, p. 335 - 354, 2004.

PIZATTO, W. Avaliação biométrica da estrutura e da dinâmica de uma Floresta Ombrófila Mista em São João do Triunfo - PR: 1995 a 1998. Curitiba, 1999. 172 p. Dissertação (Mestrado em Engenharia Florestal) - Setor de Ciências Agrárias, Universidade Federal do Paraná.

PRODAN, M.; PETERS, R.; COX, F.; REAL, P. Mensura Forestal. San José, C. R.: Deutsche Gesellschaft für Technische Zusammenarbeit (GTZ) GmbH: Instituto Interamericano de Cooperación para la Agricultura (IICA), 1997.

ROSSI, L. M. B. Processo de Difusão para Simulação da Dinâmica de Floresta Natural. 168 f. Tese (Doutorado em Engenharia Florestal) - Curso de Pós-Graduação em Engenharia Florestal, Universidade Federal do Paraná, Curitiba, 2007.

SANQUETTA, C. R.; DALLA CORTE, A. P.; EISFELD, R. L. Crescimento, mortalidade e recrutamento em duas florestas de Araucária (Araucaria angustifolia (Bert.) O. Ktze.) no estado do Paraná, Brasil. Revista Ciências Exatas e Naturais, v. 5, n. 1, Jan/Jun 2003. p. 101 - 112.

SPATHELF, P.; DURLO. M. A. Transition matrix for modeling the dynamics of a subtropical seminatural forest in southern Brazil. Forest Ecology and Management, Amsterdam, v. 151, p. 139 $149,2001$.

VANCLAY, J. K. Aggregating tree species to develop diameter increment equations for tropical rainforests. Forest Ecology and Management, Amsterdam, v. 42, p. 143 - 168, 1991.

Modeling forest growth and yield: applications to mixed tropical forests. Wallingford: $\mathrm{CAB}$ International, 1994. $312 \mathrm{p}$.

ZEIDE. B. Accuracy of equations describing diameter growth. Canadian Journal of Forest Research, Ottawa, v. 19, n. 10, p. 1283 - 1286, 1989. 